\title{
A note on new indices for the equatorial Indian Ocean oscillation
}

\author{
P A Francis ${ }^{1, *}$ and Sulochana Gadgil ${ }^{2}$ \\ ${ }^{1}$ Indian National Centre for Ocean Information Services, Ministry of Earth Sciences, Hyderabad 500 090, India. \\ ${ }^{2}$ Centre for Atmospheric and Oceanic Sciences, Indian Institute of Science, Bangalore 560 012, India. \\ *Corresponding author.e-mail: francis@incois.gov.in
}

It is now well known that there is a strong association of the extremes of the Indian summer monsoon rainfall (ISMR) with the El Niño and southern oscillation (ENSO) and the Equatorial Indian Ocean Oscillation (EQUINOO), later being an east-west oscillation in convection anomaly over the equatorial Indian Ocean. So far, the index used for EQUINOO is EQWIN, which is based on the surface zonal wind over the central equatorial Indian Ocean. Since the most important attribute of EQUINOO is the oscillation in convection/precipitation, we believe that the indices based on convection or precipitation would be more appropriate. Continuous and reliable data on outgoing longwave radiation (OLR), and satellite derived precipitation are now available from 1979 onwards. Hence, in this paper, we introduce new indices for EQUINOO, based on the difference in the anomaly of OLR/precipitation between eastern and western parts of the equatorial Indian Ocean. We show that the strong association of extremes of the Indian summer monsoon with ENSO and EQUINOO is also seen when the new indices are used to represent EQUINOO.

\section{Introduction}

The strong link between the interannual variation of Indian summer monsoon rainfall (ISMR) and El Niño and the southern oscillation (ENSO) with a higher propensity of droughts in association with El Niño and of excess rainfall seasons in association with La Niña (Sikka 1980; Rasmusson and Carpenter 1983) is well known. Recent studies have shown that, in addition to ENSO, equatorial Indian Ocean oscillation (EQUINOO) also plays an important role in the interannual variation of ISMR (Gadgil et al. 2003, 2004, 2007; Ihara et al. 2007; Francis and Gadgil 2010; Boschat et al. 2012; Rajeevan et al. 2012). A comprehensive review of the association between the EQUINOO and the regional climate over the Indian Ocean and the surrounding landmass is given in Vinayachandran et al. (2009). EQUINOO is considered to be the atmospheric component of the Indian Ocean dipole mode (IOD, Saji et al. 1999). The anomalies of convection/outgoing longwave radiation (OLR, Liebmann and Smith 1996), over the western equatorial Indian Ocean (WEIO: $50^{\circ}-70^{\circ} \mathrm{E}, 10^{\circ} \mathrm{S}-10^{\circ} \mathrm{N}$ ) tend to be of the opposite sign to the OLR anomalies over the eastern equatorial Indian Ocean (EEIO: $90^{\circ}-110^{\circ} \mathrm{E}, 10^{\circ} \mathrm{S}-\mathrm{EQ}$ ) during each of the summer monsoon months (June-September), and the correlation between the OLR of these regions is negative (figure 1a, b). EQUINOO is defined as the oscillation between a state in which the convection/precipitation is enhanced over WEIO and suppressed over the EEIO (positive phase, e.g., August 1994 (figure 1c, d)) and that in which

Keywords. Indian summer monsoon variability; equatorial Indian Ocean oscillation; Indian Ocean dipole; El Niño and southern oscillation. 

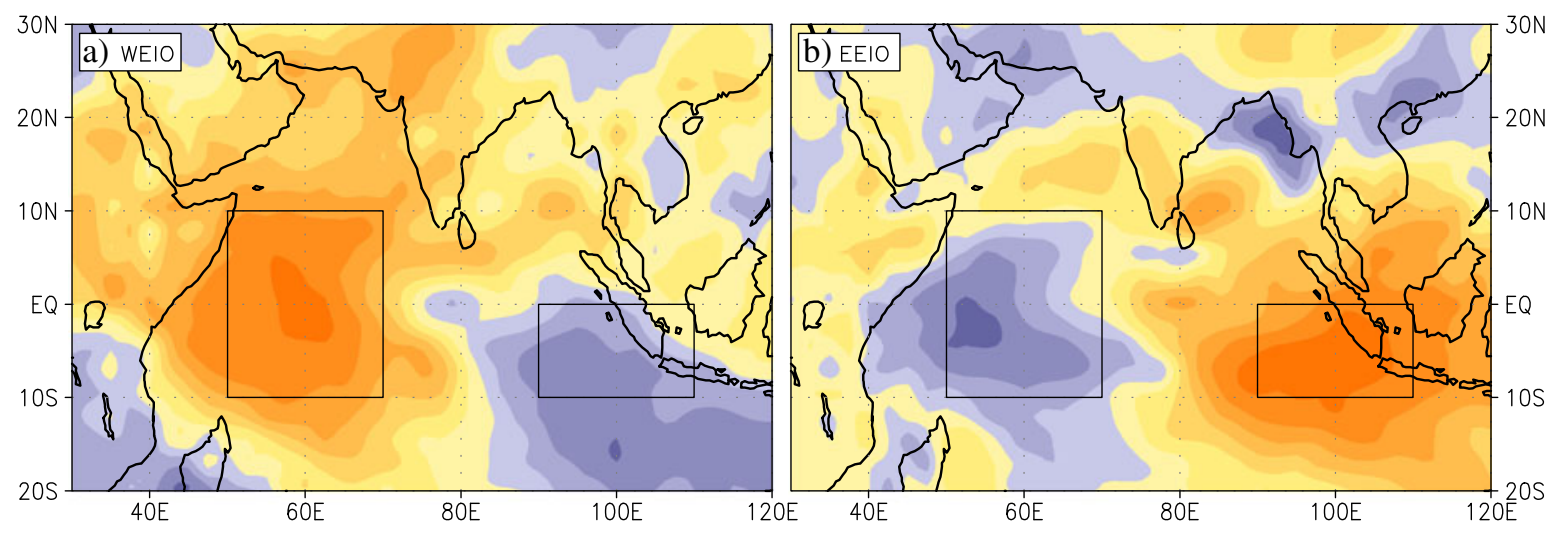

$-0.9-0.75-0.6-0.45-0.3-0.15 \quad 0 \quad 0.15 \quad 0.3 \quad 0.45 \quad 0.6 \quad 0.75 \quad 0.9$
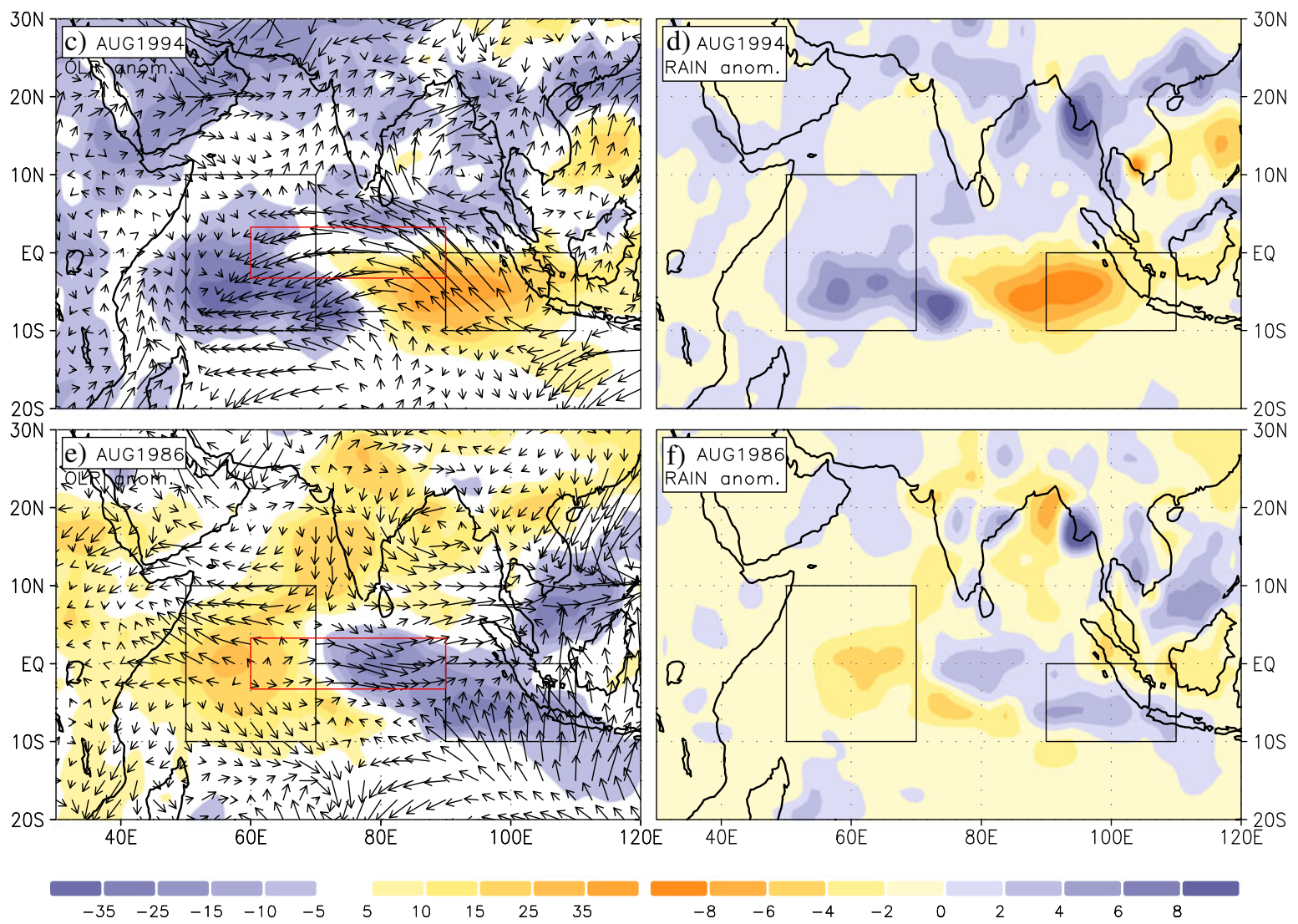

Figure 1. Correlation between OLR and OLR averaged over (a) WEIO and (b) EEIO. Anomaly patterns OLR and surface wind for (c) August 1994 and (e) August 1986. Anomaly patterns of precipitation for (d) August 1994 and (f) August 1986.

convection/precipitation is enhanced over EEIO and suppressed over WEIO (negative phase, e.g., August 1986 (figure 1e, f)).

Although the distinguishing attribute of EQUINOO is the see-saw in the convection over EEIO and WEIO, and the monsoon-EQUINOO link is manifested as a link between the OLR anomaly over the Indian region and that over the equatorial Indian Ocean, so far the index used for EQUINOO is based on the surface zonal wind over the central equatorial Indian Ocean. This was essentially due to the availability of wind data for a longer period compared to the satellite derived OLR data. Gadgil et al. (2004) used an index for EQUINOO based on the zonal component of the surface wind averaged over the central equatorial Indian Ocean (CEIO: $\left.60^{\circ}-90^{\circ} \mathrm{E}, 2.5^{\circ} \mathrm{S}-2.5^{\circ} \mathrm{N}\right)$. This zonal wind index (henceforth EQWIN) is defined as the negative of the anomaly of the surface zonal wind over CEIO, normalized by its standard deviation. It was derived from the surface wind data available from NCEP Reanalysis of 1958 (Kalney 
et al. 1996). The results of Ihara et al. (2007) who used COADS data (Worley et al. 2005) to derive the zonal wind index for a much longer period (1881-1998) to study the association of ISMR with ENSO and EQUINOO are consistent with those of Gadgil et al. (2004).

We believe that a more appropriate measure of the phase and strength of EQUINOO would be an index based on the OLR anomalies over WEIO and EEIO. An index based on OLR would also be very useful for the research community since it can be readily derived from the OLR data which are extensively analysed. For assessment of the performance of models in simulating/predicting the monsoon, generally rainfall is analysed (e.g., Rajeevan et al. 2012). Satellite derived rainfall data are also available from 1979. Hence it would also be useful to define an index of EQUINOO based on rainfall. In this note, we propose such indices for EQUINOO based on east-west gradients of OLR and rainfall anomalies.

\section{Data}

The ISMR data used here are from the archive at http://www.tropmet.res (Parthasarathy et al. 1995) and long term mean and standard deviation (for the period 1871-2011) have been used in computation of the normalized anomalies. The ENSO index used is the negative of the normalized SST anomaly averaged over the NINO3.4 region in the Pacific Ocean (NINO 3.4 SST anomaly data is taken from http://www.cpc.ncep.noaa.gov). EQWIN is based on the surface wind data from NCEP reanalysis and the long term mean and standard deviation used for the computation of EQWIN are calculated using the data for the period 1958-2011. Monthly mean OLR data is downloaded from http://www.cdc.noaa.gov and monthly mean merged rainfall data (Xie and Arkin 1996) is obtained from ftp://ftp.cpc.ncep.noaa. gov/precip/cmap/. EQUINOLR and EQUINRAIN are derived from OLR/rainfall data for the period 1979-2011.

\section{Indices of EQUINOO}

EQWIN, the index used for EQUINOO is based on the surface zonal wind over the central equatorial Indian Ocean. Surface wind over the tropical oceans is believed to be primarily driven by the heating associated with the condensation of water vapour in deep convection (Webster 1972; Gill 1980) or by the SST gradients (Lindzen and Nigam 1987). Which of the two is important depends on how much of the pressure gradient at the surface is imprinted from above by the free tropospheric pressure gradient (due to the heating gradient associated with deep convection) and how much is directly imposed by the SST (Sobel 2007). Bacmeister and Suarez (2002)'s analysis of the NCEP-NCAR reanalysis for the zonal component of the wind, showed that for the most part, the free tropospheric component dominated the SST controlled component. Chang et al.'s (2001) study for a dry linear GCM showed that in the determination of the zonal component of the wind, the free tropospheric heating gradient tended to dominate. We, therefore, expect the zonal wind over the central equatorial Indian Ocean to be primarily determined by the east-west OLR gradient over the equatorial Indian Ocean. Thus, when convection is enhanced in the western part of the equatorial Indian Ocean (positive phase of EQUINOO), the anomalous pressure gradient force is expected to be westward and anomalies in the zonal wind to be easterly over the central equatorial Indian Ocean (e.g., August 1994, figure 1c). On the other hand, when convection is enhanced over the eastern part of the equatorial Indian Ocean (negative phase of EQUINOO), westerly anomalies of the zonal wind are expected over the central equatorial Indian Ocean (e.g., August 1986, figure 1e). Anomaly of the zonal wind over the central equatorial Indian Ocean was considered as one of the measures of strength of the Indian Ocean Dipole (Saji et al. 1999).

The difference between the OLR anomalies over EEIO and WEIO is found to be highly correlated with EQWIN (coefficient 0.77) with a consistency in the sign of the two indices for a vast majority of the years (figure 2a). However, the summer monsoon of 2011 turned out to be a major exception, with a negative value of EQWIN associated with a large positive value of the difference between the OLR anomalies of EEIO and WEIO. For example, the OLR anomaly pattern for August 2011 indicates a substantiate enhancement of convection over the WEIO and suppression of convection over the EEIO. The magnitude of the anomalies is so large that the region with low OLR, which is located over EEIO in the climatology, shifts to WEIO (figure 2b). These OLR and OLR anomaly patterns are similar to those observed in August 2007 (figure 2b). In August 2007, as expected, the surface wind as well as its anomaly are easterly along the CEIO. On the other hand, in August 2011 the mean zonal wind over the CEIO is strong and westerly, which is inconsistent with the OLR pattern. As for August 2007 and 2011, for JuneSeptember 2007 and 2011 also, the convection gradients imply easterly winds over the central equatorial Indian Ocean. Furthermore, the SST 

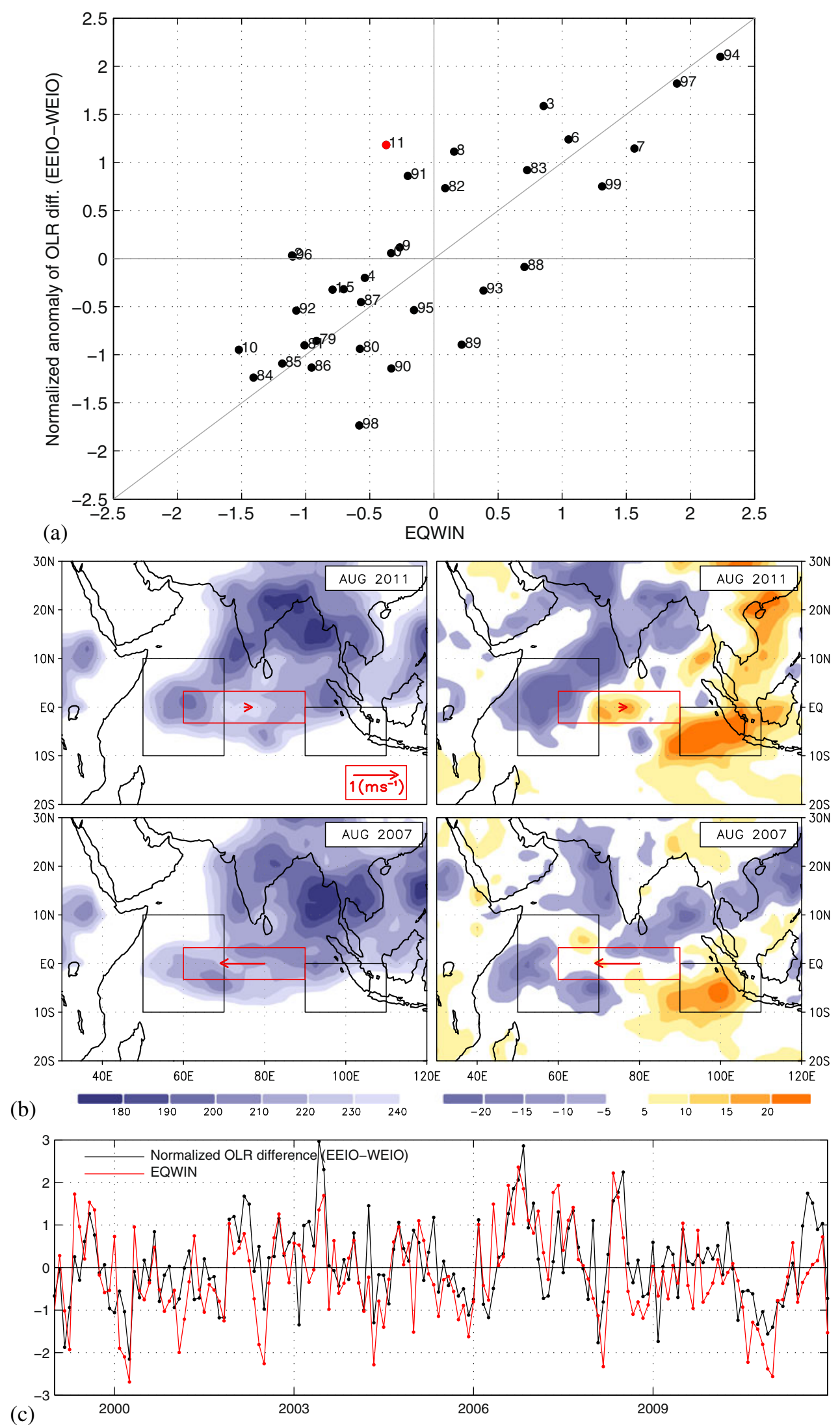

Figure 2. (a) Normalized (by standard deviation) anomaly of the difference in June-September mean OLR between EEIO and WEIO is plotted against seasonal values of EQWIN for each year in the period 1979-2011. (b) OLR (left) and OLR anomaly (right) patterns for August 2011 (top) and August 2007 (bottom). Surface zonal wind and surface zonal wind anomaly averaged over the CEIO are overlaid on the OLR and OLR anomaly patterns respectively. (c) Monthly variation of EQWIN and the difference in OLR anomaly between EEIO and WEIO for the period 1999-2011. 
gradients in all these cases also favour easterly winds. Thus the zonal surface wind as well as the anomaly are inconsistent with the OLR and SST gradients in June-September 2011.

It is important to note that the zonal component of the winds over the equatorial Indian Ocean, nearly vanishes at the equator during both monsoons (Godfrey et al. 2001; Miyama et al. 2003; Schott et al. 2009). Thus, during the summer monsoon season, the zonal wind over CEIO is weak and highly variable (mean of $0.38 \mathrm{~m} / \mathrm{s}$ and the standard deviation of $0.9 \mathrm{~m} / \mathrm{s}$ ), implying that small errors in the derived wind product could imply large errors in the value of the index. Since, the OLR is a robust product, we believe that the inconsistency in 2011 is more likely to have arisen from the wind estimates. Continuous surface wind data of sufficiently long period (more than 30 years) over the equatorial Indian Ocean are available only from re-analysis products, in which data from different sources are assimilated in the circulation models. During 1999 to 2009, the primary source of the wind data over the open ocean are scatterometer measurements from the satellite and observations from ships. With the demise of QUICKSAT in late 2009, the frequency of satellite observations has decreased considerably. We find that relative to the era with QUICKSCAT, there is a negative bias in values of EQWIN vis-a-vis the east-west anomaly of the difference in OLR (figure 2c). The small sample for the post-QUICKSAT era, precludes a systematic study of this bias at this point. We note that for the other seasons in which the surface wind as well as surface wind anomaly are inconsistent with the gradient of OLR and OLR anomaly (outliers in the wrong quadrants) are 1989, 1991 and 1993 (figure 2b). In fact, as in 2011, in all these three cases, the east-west gradients of the SST and the convection act in the same direction. As in 2011, the zonal wind and the wind anomaly over the central equatorial Indian Ocean are inconsistent with these gradients, suggesting the possibility of some problems with the quality of the surface wind data in the pre-QUICKSAT era as well.

Since the major signature of EQUINOO is the OLR/rainfall patterns, and since OLR/satellite derived rainfall data are now available for well over 30 years (1979-2011) we propose switching over to indices based on OLR/rainfall for assessing the phase and strength of EQUINOO. The index based on OLR, viz., EQUINOLR is defined as the difference between the OLR anomaly over EEIO and that over WEIO normalized by the standard deviation. Similarly the index based on rainfall, EQUINRAIN, is defined as the difference between the rainfall anomaly over the WEIO and that over the EEIO, normalized by the standard deviation. Positive (negative) values of EQUINOLR, EQUINRAIN represent a positive (negative) EQUINOO, which is favourable (unfavourable) for the ISMR.

\section{EQUINOO and the interannual variation of ISMR}

The most important finding of Gadgil et al. (2004) is the clear separation of the ISMR extremes (i.e., droughts and excess rainfall seasons) in the phase plane of the indices of the ENSO and the EQUINOO (figure 3a). They have shown that the separation is statistically highly significant, with the probability of such a separation occurring by chance being $0.157 \%$. This clear separation suggests that there is a strong relationship between the extremes of ISMR and a composite index of ENSO and EQUINOO which is the distance from the line of separation ' $\mathrm{L}$ ' (Gadgil and Francis 2012). All droughts are characterized by negative values of the index (i.e., points below the line L) and all excess rainfall seasons with positive values (i.e., points above the line L). In fact, for the extremes that occurred after the period analyzed by Gadgil et al. (2004), the composite indices of the two drought seasons (viz., 2004 and 2009) are negative and the excess season (2007) is positive (figure $3 \mathrm{a}$ ) and hence consistent with the findings of Gadgil et al. (2004). If we recalculate the probability of occurrence of such a separation by chance after including these three extreme seasons of ISMR, it is even smaller (0.024 only).

We find that in the phase planes of the ENSO index and, the EQUINOO indices based on OLR and rainfall also, there is a clear separation between droughts and excess rainfall seasons of ISMR. In figure 3(b), the ISMR is represented in the phase plane of the seasonal mean values of EQUINOLR and the ENSO index for the period 1979-2011. There are eight drought seasons (viz., 1979, 1982, 1985, 1986, 1987, 2002, 2004, 2009) and four excess seasons $(1983,1988,1994$, 2007) of Indian summer monsoon in the chosen period between 1979 and 2011. Out of these, the drought of 1985 was in the favourable phase of ENSO, while the excess season of 1994 was in the unfavourable phase. Consistent with the earlier results by Gadgil et al. (2004), all the four excess seasons are well separated from the eight drought seasons in the phase plane of the ENSO index and the proposed EQUINOLR by the line L, suggesting that a composite index based on the two indices explains the extremes of ISMR better than the individual indices. Note that the line ' $L$ ' is not unique and it can be anywhere in the 'wedge' with an angle of $13.7^{\circ}$ as shown in figure $3(\mathrm{~b})$. 

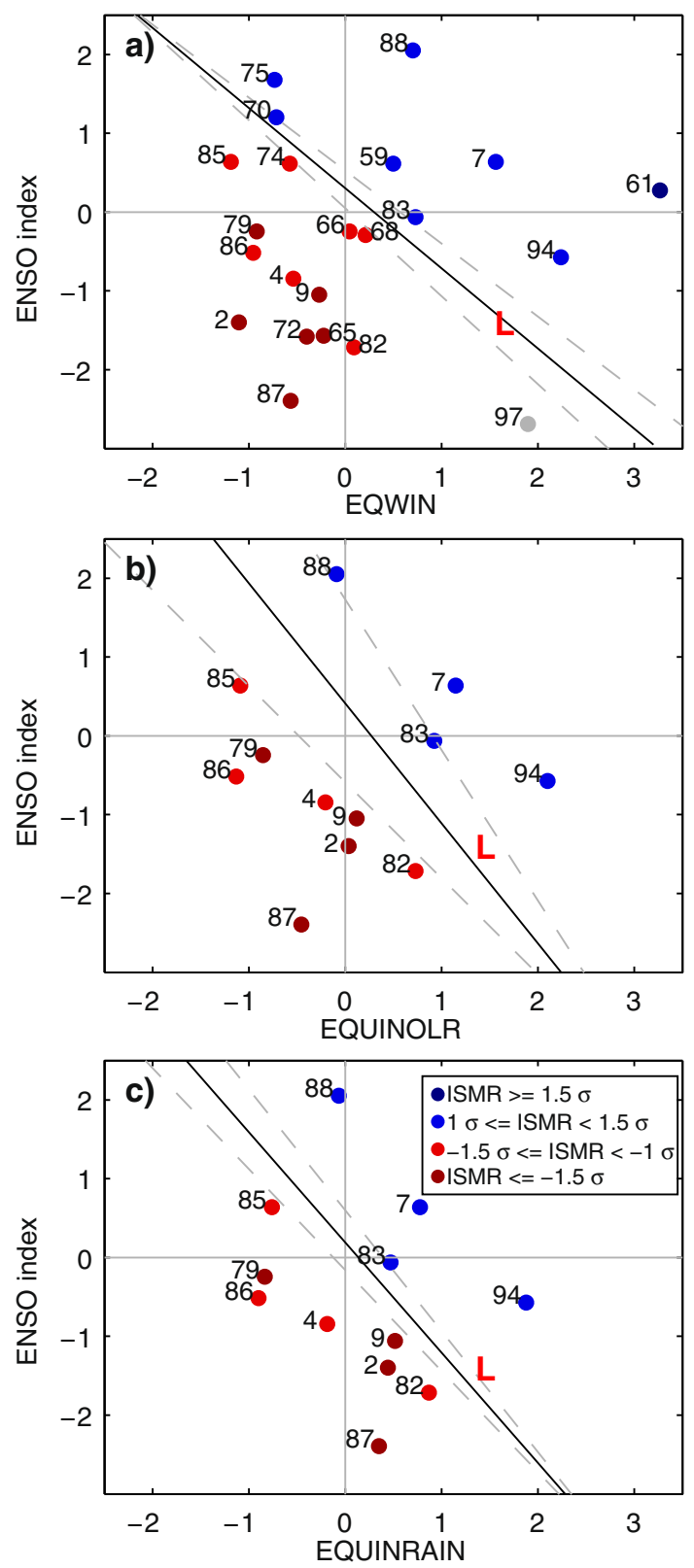

Figure 3. Extremes of ISMR in the period 1958-2011 are represented in the phase-plane of (a) EQWIN and ENSO index, (b) EQUINOLR and ENSO index for (1979-2011) and (c) EQUINRAIN and ENSO index for (1979-2011).

We construct a composite index (CI) as the linear combination of the ENSO index and the EQUINOLR in the form, $\mathrm{CI}=a^{*}$ (ENSOindex $)+$ $b^{*}$ (EQUINOLR). Here the coefficients ' $a$ ' and ' $b$ ' are taken as $\sin (\theta)=0.56$ and $(1-\sin (\theta))=0.44$ respectively where $\theta$ is the slope of the line ' $L$ ' in figure $3(\mathrm{~b})$ that separates the droughts from excess seasons. The correlation between the composite index and the ISMR is significantly higher (0.62) compared to the correlation between ISMR and the individual indices (viz., EQUINOLR and the ENSO index) for the period 1979-2011, which are 0.26 and 0.42 , respectively. The drought and excess seasons are well separated in the phase plane of EQUINRAIN and ENSO index also (figure 3c). We would therefore like to suggest that EQUINOLR and EQUINRAIN could be used for assessing the phase and magnitude of EQUINOO.

\section{Conclusions}

The anomalies of OLR,over the WEIO tend to be of the opposite sign to the OLR anomalies over the EEIO during each of the summer monsoon months (June-September). EQUINOO is defined as the oscillation between a state in which the convection/precipitation is enhanced over WEIO and suppressed over the EEIO and vice versa. The index of EQUINOO used so far is EQWIN, which is derived from the zonal wind anomaly over the central equatorial Indian Ocean, for which a long time series of data are available. We note that in the recent years, particularly after the non-availability of wind data from QuickSCAT to assimilate in the re-analysis products, there are occasions on which EQWIN is not consistent with the zonal gradient OLR anomalies. Since OLR data are more reliable than the wind over the CEIO and now there is an adequate length of satellite data, in this paper, we introduce two new indices of EQUINOO, which are defined as the difference in the anomaly of OLR/precipitation between WEIO and EEIO.

The major finding of Gadgil et al. (2004), that all the droughts in the period 1958-2004 are well separated from the excess seasons in the phase-plane of EQWIN and ENSO index, is valid even when EQUINOLR (or EQUINRAIN) is used instead of EQWIN. This shows the robust nature of EQUINOO-ISMR association, which is independent of the index chosen for defining EQUINOO. Since, the separation between the excess and drought seasons are larger in the phase plane of EQUINOLR and ENSO index compared to that in the phase plane of EQUINRAIN and ENSO index, EQUINOLR may be a more suitable index for studying the association between EQUINOO and ISMR. However, EQUINRAIN is a useful index to define EQUINOO, especially when the simulations by the climate model are analyzed to study the EQUINOO and its links with regional climate.

\section{References}

Bacmeister J T and Suarez M J 2002 Wind stress simulations and the equatorial momentum budget in an AGCM; J. Atmos. Sci. 59 3051-3073.

Boschat G, Pascal Terray and Sebastien Masson 2012 Robustness of SST teleconnections and precursory patterns associated with the Indian summer monsoon; Clim. Dynam. 38 2143-2165, doi: 10.1007/s00382-011-1100-7. 
Chiang J C, Zebiak S E and Cane M A 2001 Relative roles of elevated heating and surface temperature gradients in driving anomalous surface winds over tropical oceans; J. Atmos. Sci. 58 1371-1394.

Francis P A and Gadgil Sulochana 2010 Towards understanding the unusual Indian monsoon in 2009; J. Earth Syst. Sci. 119(4) 397-415.

Gadgil Sulochana and Francis P A 2012 Oceans and the Indian monsoon; Monsoon Monograph 2 India Meteorological Department.

Gadgil Sulochana, Rajeevan M and Francis P A 2007 Monsoon variability: Links to major oscillations over the equatorial Pacific and Indian oceans; Curr. Sci. 93(2) 182.

Gadgil Sulochana, Vinayachandran P N and Francis P A 2003 Droughts of Indian summer monsoon: Role of clouds over the Indian Ocean; Curr. Sci. 85 1713-1719.

Gadgil Sulochana, Vinayachandran P N, Francis P A and Sidharata Gadgil 2004 Extremes of the Indian summer monsoon rainfall, ENSO and the equatorial Indian Ocean Oscillation; Geophys. Res. Lett. 31(L1) 2213, doi: 10.1029/2004GL019733.

Gill A E 1980 Some simple solutions for heat induced tropical circulation; Quart. J. Roy. Meteorol. Soc. 106 $447-462$.

Godfrey J S, Johnson G C, McPhaden M J, Reverdin G and Wijffels S 2001 The tropical ocean circulation; In: Ocean Circulation and Climate (eds) Church J, Gould J and Siedler G, (Academic, London), pp. 215-224.

Ihara C, Kushnir Y, Cane M A and Pena V H D L 2007 Indian summer monsoon rainfall and its link with ENSO and Indian Ocean climate indices; Int. J. Climatol. 27 179-187.

Kalnay E et al. 1996 The NCEP/NCAR 40-year reanalysis project; Bull. Am. Meteorol. Soc. 2 437- 471.

Liebmann B and Smith C A 1996 Description of a complete outgoing longwave radiation dataset; Bull. Am. Meteorol. Soc. 77 1275-1277.

Lindzen R S and Nigam S 1987 On the role of sea surface temperature gradients in forcing low level winds and convergence in the tropics; J. Atmos. Sci. 44 2418-2436.

Miyama T, McCreary J P Jr, Jensen T G, Loschnigg J, Godfrey S and Ishida A 2003 Structure and dynamics of the Indian Ocean cross-equatorial cell; Deep-Sea Res. Part II 50 2023-2047.

Parthasarathy B, Munot A A and Kothawale D R 1995 Monthly and seasonal rainfall series for all-India homogeneous regions and meteorological subdivisions, Indian Institute of Tropical Meteorology, Pune, India, pp. 1871-1994.

Rajeevan M, Unnikrishnan C K and Preethi B 2012 Evaluation of the ENSEMBLES multi-model seasonal forecasts of Indian summer monsoon variability; Clim. Dynam. 38 2257-2274, doi: 10.1007/s00382-011-1061-x.

Rasmusson E M and Carpenter T H 1983 The relationship between eastern equatorial Pacific sea surface temperatures and rainfall over India and Sri Lanka; Mon. Weather Rev. $111517-528$.

Saji N H, Goswami B N, Vinayachandran P N and Yamagata T 1999 A dipole mode in the tropical Indian Ocean; Nature 401 360-363.

Schott F A, Xie S P and McCreary J P 2009 Indian Ocean circulation and climate variability; Rev. Geophys. 47 RG1002, doi: 10.1029/2007RG000245.

Sikka D R 1980 Some aspects of the large-scale fluctuations of summer monsoon rainfall over India in relation to fluctuations in the planetary and regional scale circulation parameters; Proc. Indian Acad. Sci. (Earth Planet. Sci.) 89 179-195.

Sobel A H 2007 Simple models of ensemble-averaged tropical precipitation and surface wind, given the sea surface temperature in the global circulation of the atmosphere, (eds) Schneider T and Sobel A H, Priceton University Press, pp. 219-252.

Vinayachandran P N, Francis P A and Rao S A 2009 Indian Ocean dipole: Processes and impacts; In: Current Trends in Science (ed.) Mukunda N, Indian Academy of Sciences, Bangalore.

Webster P J 1972 Response of the tropical atmosphere to local steady forcing; Mon. Weather Rev. 100 518-541.

Worley S J, Woodruff S D, Reynolds R W, Lubker S J and Lott N 2005 ICOADS release 2.1 data and products; Int. J. Climatol. 25 823-842.

Xie and Arkin 1996 Analyses of global monthly precipitation using gauge observations, satellite estimates, and numerical model predictions; J. Climate 9 840-858. 